\title{
Desarrollo y evaluación de modelos de predicción de Finura en vellones de vicuña, mediante espectroscopía de reflectancia en el infrarrojo cercano.
}

\section{Development and evaluation of Fineness prediction models in vicuña fleeces, by near infrared reflectance spectroscopy}

Presentación: 6-7/10/2020

\section{Doctorando:}

\section{José Ignacio Amorena}

Consejo Nacional de Investigaciones Científicas y Técnicas (CONICET) - Instituto Nacional de Tecnología Agropecuaria (INTA) Estación Experimental Agropecuaria Sumalao, Catamarca, Argentina

Amorena.jose@inta.gob.ar

\section{Director/a:}

\section{Elvira Fernández de Ahumada}

\section{Co-director/a:}

\section{Dolores María Eugenia Álvarez}

\section{Resumen}

El sistema productivo de fibra de vicuña (Vicugna vicugna) requiere de tecnologías que permitan determinar objetivamente la calidad de aquéllas. En este trabajo se desarrollaron y evaluaron modelos de predicción de la Finura Media en vellones de vicuña, mediante espectroscopía de reflectancia en el infrarrojo cercano. El modelo con mayor capacidad predictiva evidenció un coeficiente de determinación $\mathrm{R}^{2}=0,72$ y Valor Predictivo Residual=2,10. Esto da la pauta del potencial de la espectroscopía para utilizarse en determinaciones generales del parámetro evaluado.

Palabras clave: NIRS, Vicuña, Fibras Textiles, Finura Media

\begin{abstract}
The vicuña fibre (Vicugna vicugna) production system requires technologies that allow the quality of the fibers to be objectively determined. In this work, prediction models of Average Fineness in vicuña fleeces were developed and evaluated by near infrared reflectance spectroscopy. The model with the highest predictive capacity showed a determination coefficient $\mathrm{R}^{2}=0,72$ and Residual Predictive Value $=2,10$. This shows the potential of spectroscopy to be used in general determinations of the evaluated parameter
\end{abstract}

Keywords: NIRS, Vicuna, Textile Fibres, Average Fineness 


\section{Introducción}

La vicuña (Vicugna vicugna) es el más pequeño de los camélidos sudamericanos y produce una de las fibras más finas (12-14 $\mu \mathrm{m}$ ) y codiciadas del mundo (Quispe, Ramos, Mayhua, \& Alfonso, 2010). En los últimos años, diversas instituciones y organizaciones públicas y privadas han estado contribuyendo al crecimiento de la actividad textil en la provincia de Catamarca, Argentina. Tal es así que, durante el año 2019, se esquilaron 3740 animales, de los que se obtuvieron $1329 \mathrm{~kg}$ de fibra (Secretaría de Medio Ambiente, 2020). Si bien la elaboración de hilados y prendas en la provincia tiene muchos años de trayectoria, los procesos de esquila, acondicionado, tipificación y clasificación de fibra se realizaron históricamente mediante técnicas artesanales. En este sentido, la producción de fibra de vicuña requiere indicadores que reemplacen las apreciaciones subjetivas por parte de los responsables de su clasificación a los fines de la determinación de los estándares de calidad. El diámetro de la fibra, o finura, es el atributo de mayor incidencia en el precio, ya que se relaciona con la sensación subjetiva de suavidad o "mano" (Frank, Hick, Castillo, Prieto, \& Adot, 2014). Sin embargo, existen otros parámetros que también aportan a la valoración de la fibra, como el color, resistencia al quiebre, factor de picazón, rendimiento al lavado o presencia de fibras meduladas (Adot \& Frank, 2015). A fin de medir estas variables, la industria textil ha desarrollado un sinfín de técnicas (Sommerville, 2007). No obstante, la posibilidad de utilizarlas de manera habitual se torna difícil y costosa, ya que no se cuenta con laboratorios especializados en la región. La espectroscopía de reflectancia en el infrarrojo cercano (NIRS por sus siglas en inglés, Near Infrared Reflectance Spectroscopy), es una tecnología versátil, que tiene el potencial para ser utilizada en la determinación de los parámetros de calidad de las fibras (Canaza-Cayo, Alomar, \& Quispe, 2013; Zoccola, Lu, Mossotti, Innocenti, \& Montarsolo, 2013). Bajo este supuesto se lleva a cabo la tesis doctoral "Desarrollo de metodología NIRS para la evaluación cuantitativa y cualitativa de fibras especiales de origen animal", en el Laboratorio de Aceites y Grasas perteneciente al Instituto Nacional de Tecnología Agropecuaria (INTA), sede Catamarca. En los primeros ensayos se determinaron los parámetros de medición (Amorena, Álvarez, Rigalt, \& Fernández de Ahumada, 2018). Luego se desarrollaron modelos de predicción de Finura Media como parámetro de calidad en fibras de llama (Amorena, Fernández de Ahumada, Álvarez, \& Rigalt, 2019). El presente trabajo tiene como objetivo el desarrollo y evaluación del desempeño de modelos de predicción de la Finura Media en vellones de vicuña.

\section{Desarrollo}

Se recolectaron 122 muestras de vicuña de ambos sexos, de entre 1 y 6 años de edad. Éstas fueron tomadas del costillar medio del animal, donde la Finura Media presenta mayor uniformidad. Las muestras se obtuvieron en esquilas realizadas en el año 2017 en la Estación Experimental Agropecuaria INTA de Abra Pampa (Jujuy) y en la localidad de Laguna Blanca (Catamarca). Se determinó Diámetro o Finura Media (FM) y su Desvío Estándar (DE), en micrones ( $\mu$ m); el Coeficiente de Variación (CV) y el Factor de Confort (FC), que representa el porcentaje de fibras causantes de la picazón en las prendas. Tales determinaciones se realizaron en el Laboratorio de Fibras Textiles de INTA en Bariloche, Río Negro con el instrumento Optic Fiber Diameter Analyzer 2000. Las muestras fueron escaneadas en un espectrómetro NIRS DS 2500 (FOSS Analytical Systems), previamente dispuestas en celdas circulares de $50 \mathrm{~mm}$ de diámetro y $10 \mathrm{~mm}$ de profundidad. Cada una fue analizada sin y con cardado (peinado realizado con cepillo de carda). Los espectros se obtuvieron por reflectancia en la región visible (VIS) e infrarrojo cercano (NIR) (400 a $2500 \mathrm{~nm}$ ) a intervalos de 0,5 nm, contabilizando 4200 datos de absorbancia por espectro. Los espectros se almacenaron como unidades de absorbancia $(A=\log 1 / R)$, donde $\mathrm{R}$ corresponde a la energía reflejada. El espectro final de cada muestra fue el resultado del promedio de escanear la misma tres veces consecutivas en distintas posiciones dentro de la celda.

Se empleó el software WinISI (ver. 4.10; Infrasoft International). para operar el espectrómetro, recolectar los espectros y para el manejo y análisis de los datos. Los métodos de corrección de scattering o dispersión de la luz utilizados fueron Weighted Multiplicative Scatter Correction (WMSC) y Standard Normal Variate and Detrend (SNVD) (Rinnan, Berg, \& Engelsen, 2009). Los tratamientos derivativos empleados fueron: 0-0-1-1, 1-5-3-1 y 2-5-3-1. El primer dígito indica el orden de la derivada; el segundo, la cantidad de términos de la ecuación de derivación y los últimos dos, el número de términos usados para suavizar la curva. En el ensayo se aplicaron los tratamientos derivativos con y sin tratamientos de corrección de scattering (STCS). Previo al desarrollo de los modelos de predicción, se aplicaron combinaciones entre los pre-tratamientos mencionados y los espectros en el rango completo VIS-NIR (4200 datos) o la fracción NIR (2800 datos). Para el desarrollo de los modelos se utilizó el método de regresión Modified-Partial Least Squares (M-PLS) (Alomar, 
Alarcón Buhofer, \& Kusanovic, 2015). En todos los casos, se utilizó la técnica leave-one-outpara llevar a cabo la Validación Cruzada. La selección de muestras para la Validación Externa se realizó en base al valor H de Mahalanobis. Se armaron dos grupos según el número de muestras para la calibración $(n=81$ o $n=91)$ y la validación $(n=41$ o $n=31)$. La capacidad predictiva de cada modelo se evaluó en base al coeficiente de determinación R²; Error de la Validación Cruzada (SECV, Standard Error of Cross Validation); Error de la Validación Externa (SEV, Standard Error of Validation) y Valor Predictivo Residual (RPD, Residual Predictive Value). En este sentido, un modelo con RPD mayor a 3 se considera adecuado para fines analíticos, mientras que valores entre 2 y 3 indican que el modelo tiene menor capacidad predictiva y podría utilizarse para clasificaciones generales (Gishen \& Cozzolino, 2007).

\section{Resultados}

En la tabla 1 se muestran los valores correspondientes a los parámetros estadísticos de las variables de calidad analizadas. Se observa que el valor máximo $(47,94 \mu \mathrm{m})$, correspondiente a Finura Media es extremadamente alto en comparación a los valores reportados por Quispe, Rodríguez, Iñiguez, \& Mueller (2009). Sin embargo, se consideran de importancia ya que aportan a la variabilidad, la cual debería ser tan grande como la esperada en cualquier muestra futura.

\begin{tabular}{|c|c|c|c|c|c|}
\hline Parámetro & Promedio & D. E. & Mín. & Máx. & C.V. \\
\hline FM $(\mu \mathrm{m})$ & 13,85 & 3,35 & 11,31 & 47,94 & 24,19 \\
\hline $\mathrm{DE}(\mu \mathrm{m})$ & 6,58 & 2,49 & 3,32 & 25,49 & 37,82 \\
\hline $\mathrm{CV}(\%)$ & 47,09 & 10,83 & 25,3 & 78,5 & 23 \\
\hline FC $(\%)$ & 97,79 & 6,18 & 31,3 & 99,8 & 6,32 \\
\hline
\end{tabular}

Tabla 1: Indicadores estadísticos de los parámetros de calidad de muestras de fibra de vicuña. FM= Finura Media; $\mathrm{DE}=$ Desvío Estándar de la Finura Media; CV= Coeficiente de Variación (DS/FM) x 100; FC= Factor de Confort

En la figura 1 se muestran las curvas espectrales obtenidas $(\mathrm{n}=122)$. En el segmento de la región VIS (400-1100 nm) se puede observar la dispersión de las curvas debida a los colores típicos del pelaje de la vicuña que se encuentran entre el blanco y la gama del marrón. Los espectros más separados del conjunto corresponden a las muestras con valores extremos de Finura.

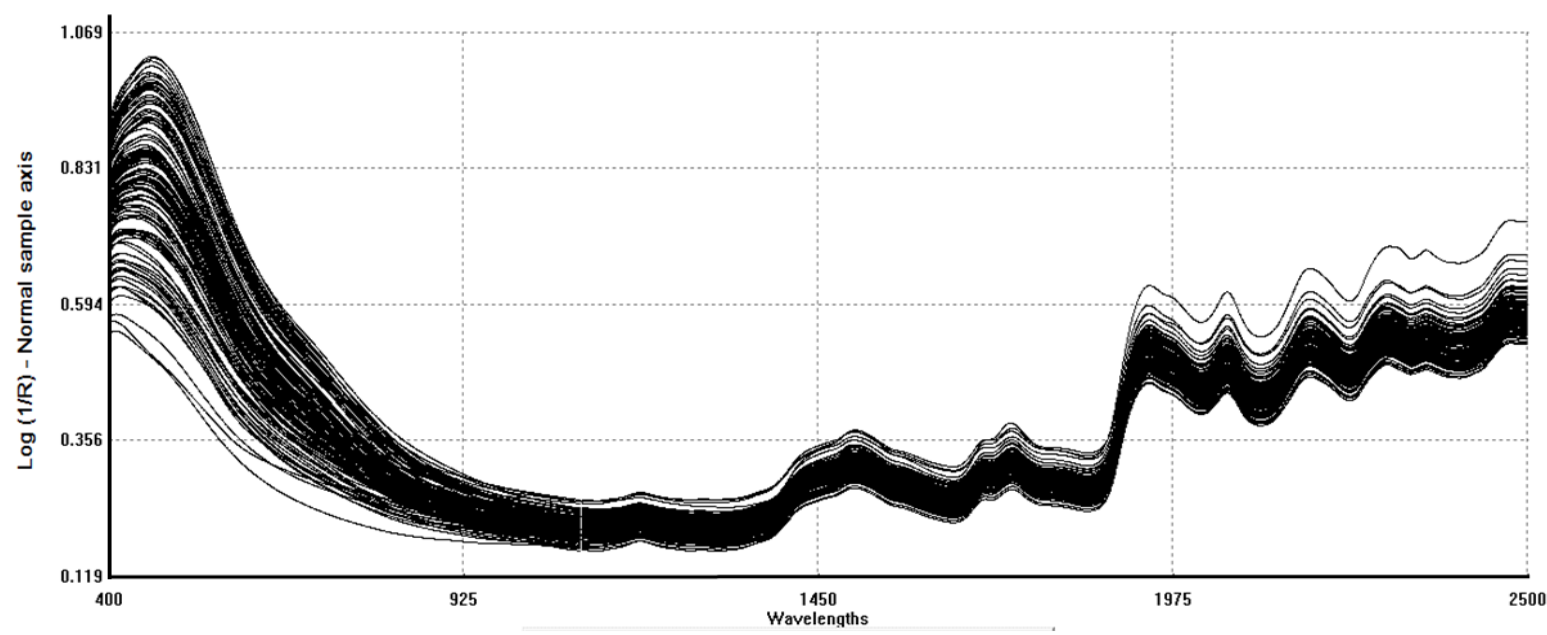

Figura 1: Espectros de las muestras de fibra de vicuña en la región VIS-NIR. Absorbancia (log 1/R) en función de longitud de onda (400-2500nm).

El espectro promedio y desvío estándar de los datos de absorción para el espectro completo VIS y NIR (400 a $2500 \mathrm{~nm}$ ) se muestran en la figura 2. El espectro promedio (eje Y izquierdo), muestra bandas de absorción en la región NIR a 1500 y $1740 \mathrm{~nm}$ correspondientes a los primeros armónicos de estiramiento de los grupos $\mathrm{N}-\mathrm{H}$ y $\mathrm{S}-\mathrm{H}$, respectivamente. Además, se observaron bandas de absorción alrededor de $1940 \mathrm{~nm}$, correspondientes a los segundos armónicos del grupo $\mathrm{OH}$, relacionados con el contenido de humedad de las fibras. Asimismo, se observaron picos de absorción a 2170 y 2280 $\mathrm{nm}$, relacionados con la deformación y combinación de grupos $\mathrm{C}-\mathrm{H}$ asociados a aminoácidos. La banda de absorción 
alrededor de los $2055 \mathrm{~nm}$ puede estar relacionada con la combinación de grupos $\mathrm{N}-\mathrm{H}$ presentes en los compuestos proteicos de las fibras (Canaza-Cayo et al., 2013). El desvío estándar del espectro (eje Y derecho), muestra valores prominentes en el rango VIS, explicados por las diferencias entre los colores mencionados anteriormente. En el rango NIR, se pueden notar variaciones más pequeñas en algunos puntos, fundamentalmente relacionadas con las principales bandas de absorción en el espectro promedio (Canaza-Cayo et al., 2013).

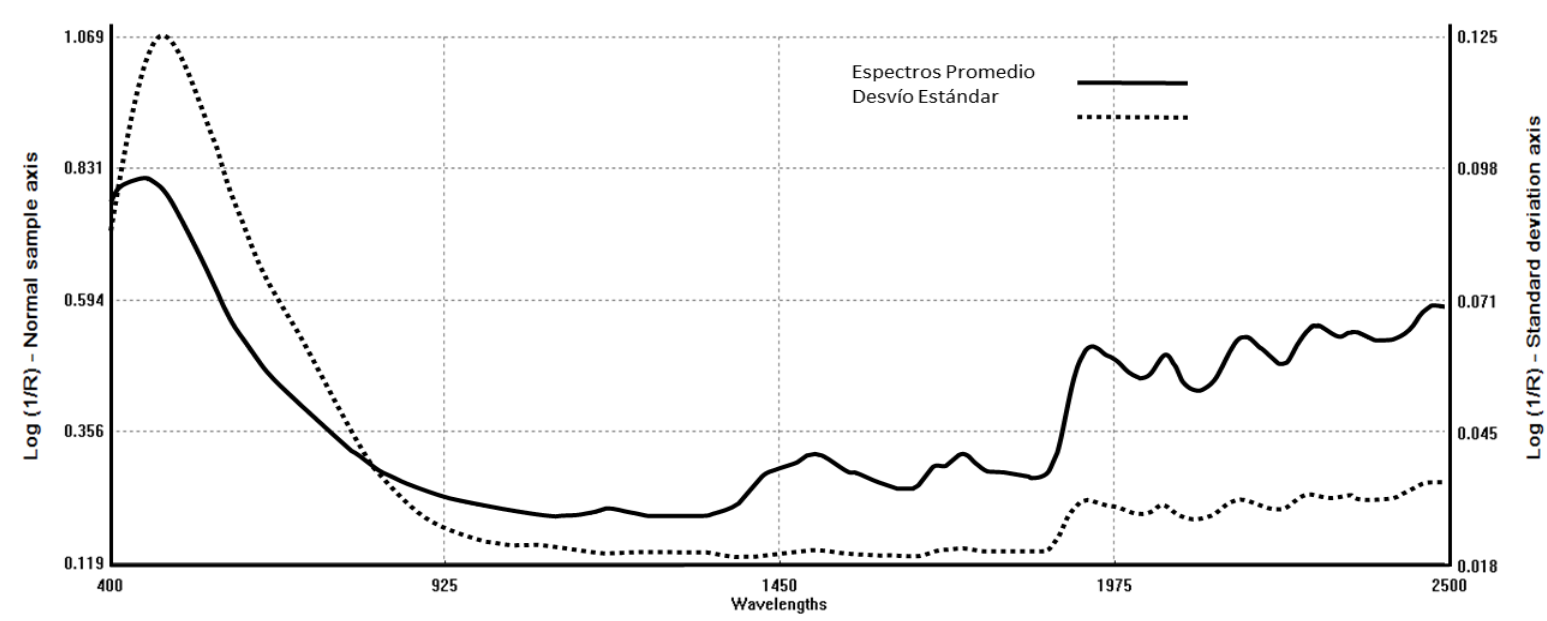

Figura 2: Espectro promedio (eje Y derecho) y desviación estandar (eje Y izquierdo) de los datos de absorción de las muestras de fibra de vicuña. Absorbancia ( $\log 1 / R)$ en función de longitud de onda (400-2500nm).

En la tabla 2 se presentan los modelos de predicción para Finura Media. Las mejores calibraciones se obtuvieron con el mínimo o ningún pre-tratamiento espectral en las muestras.

\begin{tabular}{|c|c|c|c|c|c|c|c|c|c|}
\hline Variable & Tratamiento & $\begin{array}{c}\mathbf{N} \\
\text { cal }\end{array}$ & $\begin{array}{c}\mathbf{N} \\
\text { val }\end{array}$ & $\begin{array}{l}N^{\circ} \text { de } \\
\text { datos }\end{array}$ & $\begin{array}{c}\text { Tratamientos } \\
\text { espectrales }\end{array}$ & $\mathbf{R}^{2}$ & $\begin{array}{l}\text { SECV } \\
(\mu \mathrm{m})\end{array}$ & $\begin{array}{c}\text { SEV } \\
(\mu \mathrm{m})\end{array}$ & RPD \\
\hline \multirow{10}{*}{$\begin{array}{c}\text { Finura } \\
\text { Media } \\
(\mu \mathrm{m})\end{array}$} & \multirow{5}{*}{ Sin Cardar } & 91 & 31 & 4200 & stcs $0-0-1-1$ & 0,61 & 0,731 & 0,621 & 1,71 \\
\hline & & 81 & 41 & 4200 & stcs $0-0-1-1$ & 0,57 & 0,732 & 1,033 & 1,71 \\
\hline & & 81 & 41 & 2800 & stcs $0-0-1-1$ & 0,56 & 0,727 & 1,080 & 1,72 \\
\hline & & 91 & 31 & 2800 & stcs $0-0-1-1$ & 0,54 & 0,818 & 0,744 & 1,53 \\
\hline & & 91 & 31 & 4200 & snvd $0-0-1-1$ & 0,53 & 0,755 & 1,107 & 1,66 \\
\hline & \multirow{5}{*}{ Cardado } & 91 & 31 & 4200 & stcs $0-0-1-1$ & 0,75 & 0,608 & 0,728 & 2,06 \\
\hline & & 81 & 41 & 4200 & stcs $0-0-1-1$ & 0,72 & 0,595 & 0,757 & 2,10 \\
\hline & & 91 & 31 & 2800 & stcs $0-0-1-1$ & 0,72 & 0,607 & 0,654 & 2,06 \\
\hline & & 91 & 31 & 2800 & stcs $1-5-3-1$ & 0,72 & 0,633 & 0,726 & 1,98 \\
\hline & & 91 & 31 & 4200 & stcs $1-5-3-1$ & 0,71 & 0,652 & 0,744 & 1,92 \\
\hline
\end{tabular}

Tabla 2: Parámetros estadísticos de las mejores calibraciones obtenidas para la predicción de Finura Media en fibra de vicuña. $\mathrm{N}$ cal: muestras usadas para el modelo de calibración, $\mathrm{N}$ val: muestras usadas para la validación externa, R2: coeficiente de determinación, SECV: error estándar de la validación cruzada, SEV: error estándar de la validación externa, RPD: valor predictivo residual.

Resultados similares se encontraron en ensayos realizados con fibra de llama (Amorena et al., 2019), y alpaca (CanazaCayo et al., 2013). Si bien la función del pre-tratamiento espectral es la eliminación de los fenómenos físicos que generan distorsión o ruido en los espectros, estos recursos no siempre mejoran la exactitud del modelo (Shenk, Workman, \& Westerhaus, 2008) . En tal sentido, Zoccola (2013) menciona que este comportamiento es consecuencia del espesor y morfología de las fibras, lo que contribuye a la identificación de las mismas. Otro punto a destacar es la mayor capacidad predictiva que se obtuvo con los modelos desarrollados a partir de muestras a las cuales se les realizó el tratamiento de cardado ( $\mathrm{R}^{2}>0,71$ y $\left.\mathrm{RPD}>1,92\right)$, en comparación a las muestras sin cardar $\left(\mathrm{R}^{2}<0,62\right.$ y $\left.\mathrm{RPD}<1,71\right)$. Esto podría deberse a que el cardado desenreda el vellón y a su vez le quita impurezas, lo que aumentaría la repetibilidad espectral de las 
muestras. Cabe destacar que, en ensayos previos sobre repetibilidad en vellones de vicuña, no se encontraron diferencias significativas entre muestras cardadas y sin cardar (Amorena, Álvarez, et al., 2018). Comparativamente, los resultados del modelo que presentó mayor coeficiente de correlación $\left(R^{2}=0,71\right)$ fue menor a los obtenidos en fibra de alpaca $\left(R^{2}=0,9\right)$ (Canaza-Cayo et al., 2013; Gishen \& Cozzolino, 2007), y lana de oveja limpia, $\left(\mathrm{R}^{2}=0,90\right)$; pero no así en lana sucia $\left(\mathrm{R}^{2}=\right.$ 0,50) (Cozzolino et al., 2005). El Error de Validación Cruzada más bajo de los modelos (SECV=0,595), fue considerablemente menor a los reportados en alpaca por Canaza-Cayo, et al. (2013) y Gishen \& Cozzolino, (2007) (SECV=1,06 $\mu$ m y 2,62 $\mu$ m respectivamente)(Alomar et al., 2015; Canaza-Cayo et al., 2013; Cozzolino et al., 2005; Gishen \& Cozzolino, 2007). Finalmente, el valor de RPD más alto de todos los modelos evaluados (RPD=2,10), fue menor a 3.

\section{Conclusiones}

El desempeño general de los modelos obtenidos da una idea de su capacidad para ser utilizados en clasificaciones generales de la Finura Media en vellones de vicuña. Además, se destaca que, el tratamiento de cardado del vellón le confiere al modelo una mayor capacidad predictiva, así como la nula o mínima aplicación de pre-tratamientos espectrales.

\section{Referencias}

Adot, O. G., \& Frank, E. N. (2015). Industrialization and commercialisation of the fibre of south american camelids in Argentina. International Journal of Scientific Research and Innovative Technology, 2(1), 52-59.

Alomar, D., Alarcón Buhofer, M. M. I., \& Kusanovic, A. (2015). Predicción de la calidad de lana mediante Espectroscopía de Reflectancia en el Infrarrojo Cercano (NIRS). Agro Sur, 43(1), 19-24.

Amorena, J. I., Álvarez, D. M. E., Rigalt, F., \& Fernández de Ahumada, E. (2018). Desarrollo preliminar de metodología NIRS para la evaluación cuantitativa y cualitativa de fibra de vicuña (Vicugna vicugna). 3er Congreso de Ingeniería de Procesos y Productos, (1), 241-250.

Amorena, J. I., Fernández de Ahumada, E., Álvarez, D. M. E., \& Rigalt, F. (2019). Desarrollo de modelos NIRS de predicción para el análisis de la finura de fibras textiles de vicuña y llama. En M. M. Marciszak \& O. A. Anunziata (Eds.), Jornadas de Intercambio y Difusión de los Resultados de Investigaciones de los Doctorandos en Ingeniería.

Canaza-Cayo, A. W., Alomar, D., \& Quispe, E. (2013). Prediction of alpaca fibre quality by near-infrared reflectance spectroscopy. Animal, 7(7), 1-7.

Cozzolino, D., Montossi, F., \& San Julian, R. (2005). The use of visible (VIS) and near infrared (NIR) reflectance spectroscopy to predict fibre diameter in both clean and greasy wool samples. Animal Science, 80(03), 333-337.

Frank, E. N., Hick, M. V. H., Castillo, M. F., Prieto, A., \& Adot, O. G. (2014). Fibre-based components determining handle/skin comfort in fabrics fade from dehaired and non dehaired llama fibre. International Journal of Applied Science and Technology, 4(3), 51-66.

Gishen, M., \& Cozzolino, D. (2007). Feasibility study on the potential of visible and near infrared reflectance spectroscopy to measure alpaca fibre characteristics. Animal, 1(6), 899-904.

Quispe, E. C., Ramos, H., Mayhua, P., \& Alfonso, L. (2010). Fibre characteristics of vicuña (Vicugna vicugna mensalis). Small Ruminant Research, 93(1), 64-66.

Quispe, E. C., Rodríguez, T. C., Iñiguez, L. R., \& Mueller, J. P. (2009). Producción de fibra de alpaca, llama, vicuña y guanaco en Sudamérica. Animal Genetic Resources Information, 45, 1-14.

Rinnan, Å., Berg, F. van den, \& Engelsen, S. B. (2009). Review of the most common pre-processing techniques for nearinfrared spectra. TrAC - Trends in Analytical Chemistry, 28(10), 1201-1222.

Secretaría de Medio Ambiente, C. (2020). Catamarca, una de las provincias principales en producción de fibra de vicuñas. Retrieved August 20, 2020, de https://www.portal.catamarca.gob.ar/noticias/catamarca-una-de-las-provinciasprincipales-en-produccion-de-fibra-de-vicunas-6599/ 
Shenk, J. S., Workman, J., \& Westerhaus, M. O. (2008). Application of NIR spectroscopy to agricultural products. In D. A. Burns \& E. W. Ciurczak (Eds.), Handbook of Near-Infrared Analysis (Tercera Ed, p. 811). Boca Ratín, Florida: CRC Press Taylor \& Francis Group.

Sommerville, P. (2007). Fundamental Principles of Fibre Fineness Measurement. Kensington, Australia: Australian Wool Testing Authority Ltd.

Zoccola, M., Lu, N., Mossotti, R., Innocenti, R., \& Montarsolo, A. (2013). Identification of wool, cashmere, yak, and angora rabbit fibers and quantitative determination of wool and cashmere in blend: A near infrared spectroscopy study. Fibers and Polymers, 14(8), 1283-1289. 